\title{
Sommaire
}

Remerciements .................... $\mathrm{xv}$

Liste des auteurs $\ldots \ldots \ldots \ldots \ldots \ldots \ldots \ldots$ xvii

Préface $\ldots \ldots \ldots \ldots \ldots \ldots \ldots \ldots \ldots \ldots \ldots \ldots \ldots \ldots$ xxi

\section{Chapitre 1 : Introduction}

1. Intérêt de la diffraction pour la caractérisation des contraintes et de la texture ............................. 1

2. Brève histoire de la mesure des contraintes au moyen de la technique

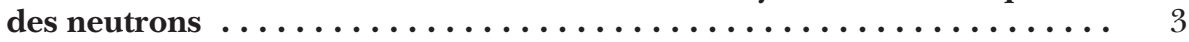

3. Portée de l'ouvrage $\ldots \ldots \ldots \ldots \ldots \ldots \ldots \ldots \ldots \ldots \ldots \ldots \ldots \ldots$

\section{Chapitre 2 : Intérêt des neutrons dans la caractérisation des matériaux}

1. Caractéristiques du neutron .................... 13

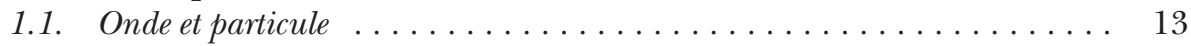

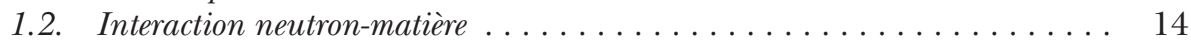

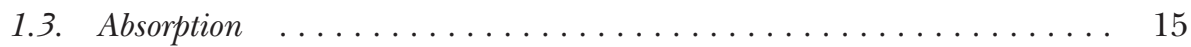

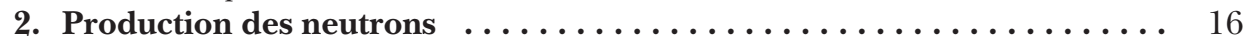

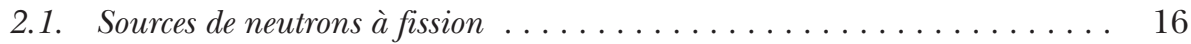

2.2. Sources de neutrons à spallation . . . . . . . . . . . . . . . 17

2.3. État des lieux et évolution future des sources de neutrons . . . . . . . . . . 18

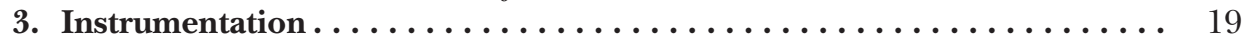

3.1. Fonction de diffusion d'un ensemble d'atomes . . . . . . . . . . . . . . 19

3.2. Diffractomètres pour poudres et mesures de déformations . . . . . . . . . 21

3.3. Comparaison entre les instruments sur sources continues
et sur sources pulsées $\ldots \ldots \ldots \ldots \ldots \ldots \ldots \ldots \ldots \ldots \ldots \ldots \ldots \ldots \ldots \ldots \ldots \ldots$

4. Principales applications de la diffusion de neutrons en science

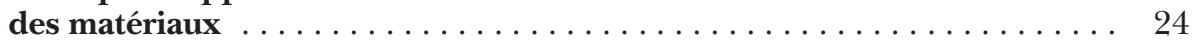

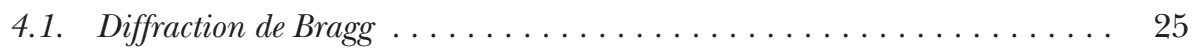




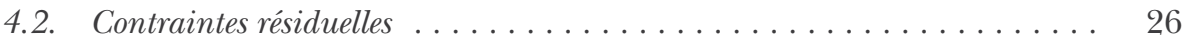

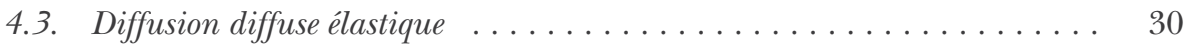

4.4. Diffusion de neutrons aux petits angles (DNPA) . . . . . . . . . 31

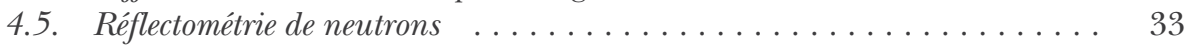

4.6. Diffusion inélastique de neutrons .................. 34

4.7. Diffusion quasi élastique de neutrons . . . . . . . . . . . . . 36

5. Autres techniques de caractérisation des matériaux utilisant

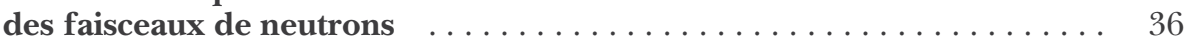

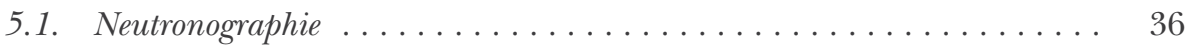

5.2. Analyse par activation neutronique $(A A N) \ldots \ldots \ldots \ldots \ldots$

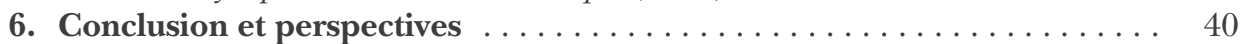

\section{Chapitre 3 : Utilisation du rayonnement synchrotron en science des matériaux}

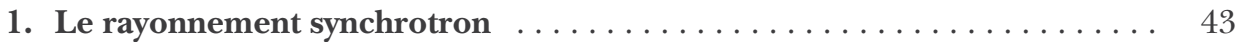

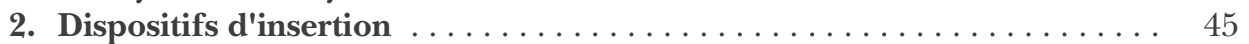

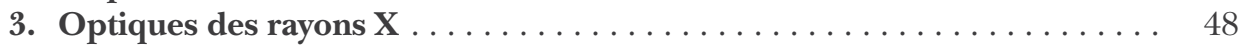

4. Diffraction en science des matériaux $\ldots \ldots \ldots \ldots \ldots \ldots \ldots \ldots$

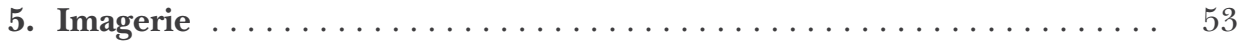

6. Diffraction et microtomographie couplées en science des matériaux .. 54

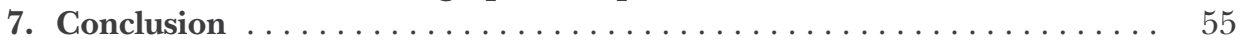

\section{Chapitre 4 : Évaluation et problèmes dans la détermination des contraintes}

\subsection{Détermination des contraintes macroscopiques par la technique de la diffraction}

1. Principe de détermination des contraintes par diffraction $\ldots \ldots \ldots \ldots 60$

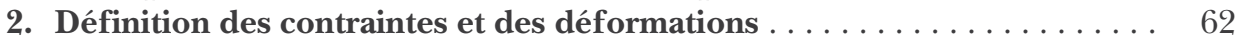

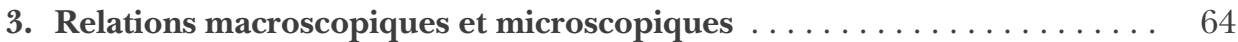

3.1. Cas d'une structure monocristalline . . . . . . . . . . . . . . . . . . . . . 64

3.2. Cas d'une structure isotrope polycristalline non texturée . . . . . . . . . . 67

3.3. Cas d'une structure polycristalline polyphasée non texturée . . . . . . . . 67

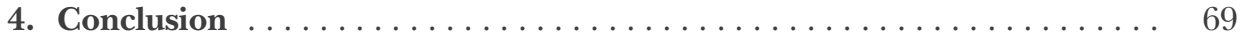

\subsection{Mesure des macrocontraintes par diffraction dans les matériaux texturés}

1. Introduction 
2. Calcul des constantes élastiques de diffraction pour

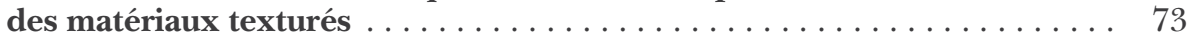

2.1. Modèles de calcul des constantes élastiques de diffraction . . . . . . . . . 74

2.2. L'approche quasi isotrope . . . . . . . . . . . . . . . . . . 80

3. Modélisation et détermination expérimentale des constantes élastiques

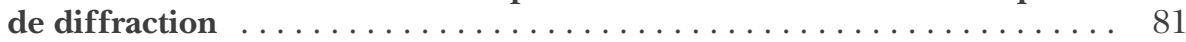

3.1. Influence de l'anisotropie du cristal ................. 81

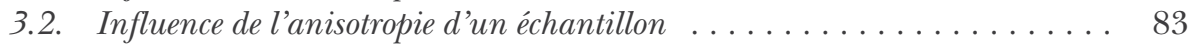

3.3. Vérification expérimentale des constantes élastiques de diffraction . . . . . . 86

4. Méthode des réflexions multiples pour la détermination des contraintes

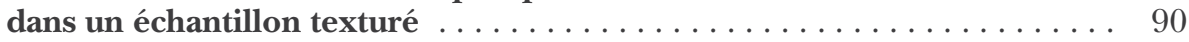

4.1. Mesures de macrocontraintes dans des échantillons de cuivre laminés à froid 92

4.2. Évolution de la microstructure et de l'état de la contrainte pendant le recuit du cuivre laminé à froid . . . . . . . . . . . . . . . . . 94

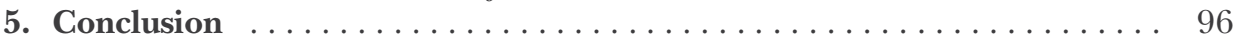

\subsection{Détermination des contraintes microscopiques par la technique de diffraction}

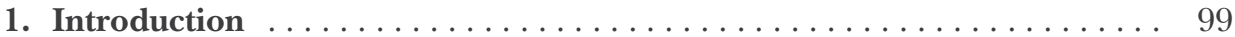

2. Approche physique et micromécanique du matériau . . . . . . . . . 100

3. Traitement des pics de diffraction et soustraction des effets instrumentaux . . . . . . . . . . . . . . . . . . . . . . 103

4. Influence des hétérogénéités de déformations élastiques des cristallites . . 105

5. Effet de la taille des domaines cohérents de diffraction et de la distribution des microdéformations d'ordre III . . . . . . . . . . . 109

6. Expression globale de l'élargissement des pics de diffraction . . . . . . . 111

\section{Chapitre 5 : Techniques de mesures}

\subsection{Diffraction des rayons $X$ de laboratoire}

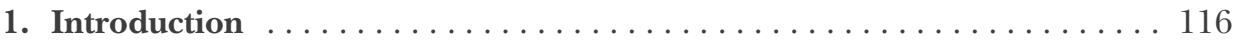

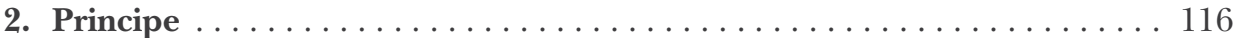

2.1. Relation entre la diffraction et la déformation élastique . . . . . . . . . . . 116

2.2. Relation entre la déformation élastique et la contrainte . . . . . . . . . . . 117

2.3. Détermination des contraintes par diffraction . . . . . . . . . . . . . 119

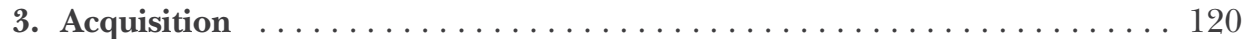

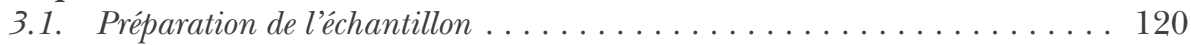

3.2. Choix des conditions de diffraction . . . . . . . . . . . . . . 121

3.3. Stratégies d'acquisition . . . . . . . . . . . . . . . . . . . 122

3.4. Réglage du goniomètre . . . . . . . . . . . . . . . . . . . . . . . 134

3.5. Problèmes de taille de cristallites . . . . . . . . . . . . . . . . . . . . . 137

3.6. Problème des pics tronqués . . . . . . . . . . . . . . . . . . . 138 
3.7. Détermination de gradients de contrainte selon la profondeur . . . . . . . . . 139

3.8. Détermination de gradients de contrainte selon la surface . . . . . . . . . . . 142

4. Traitement des données . . . . . . . . . . . . . . . . . . . . . . . . . . . . 142

4.1. Traitement des diffractogrammes . . . . . . . . . . . . . . . . . 142

4.2. Traitement des positions des pics de diffraction . . . . . . . . . . . . . . . 143

4.3. Traitement simultané de l'ensemble des données . . . . . . . . . . . . . . . . 149

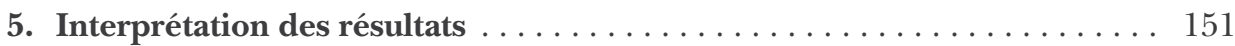

5.1. Définition du mesurande . . . . . . . . . . . . . . . . 151

5.2. Contraintes d'ordre II . . . . . . . . . . . . . . . . . . . . . . . . 152

5.3. Texture cristalline . . . . . . . . . . . . . . . . . . . . . . . . . 153

5.4. Multiphasage ......................... 155

5.5. Rugosité ............................. 155

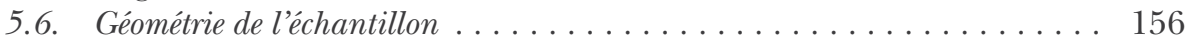

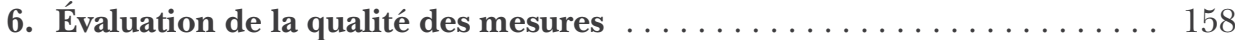

6.1. Évaluation qualitative . . . . . . . . . . . . . . . . . . . . . . . . . . . . . . . . . . 158

6.2. Calcul d'incertitude . . . . . . . . . . . . . . . . . . . . . 159

6.3. Échantillons de référence . . . . . . . . . . . . . . . . . . . . 164

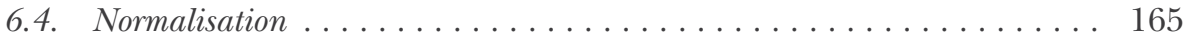

6.5. Informations nécessaires à l'évaluation de la qualité

7. Conclusion ............................... 166

\subsection{Imagerie des champs de déformation obtenue par diffraction neutronique}

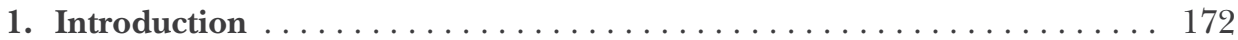

2. Spécifications pour un système d'imagerie des déformations . . . . . . 173

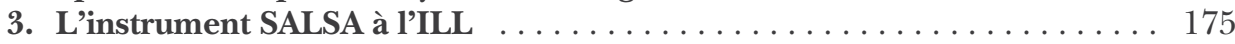

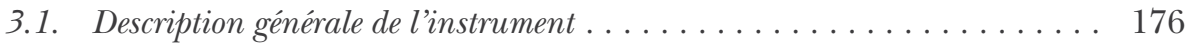

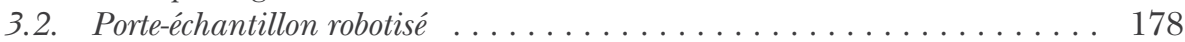

3.3. Monochromateur et guide de neutrons . . . . . . . . . . . . . . . . 182

3.4. Fentes optiques . . . . . . . . . . . . . . . . . . . 186

3.5. Collimateurs radiaux . . . . . . . . . . . . . . . . . . . . . . . 188

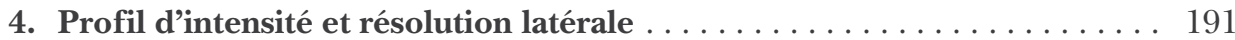

5. Imagerie et distribution de longueurs d'ondes de la sonde de mesure . . 192

6. Conclusion ................................ 197

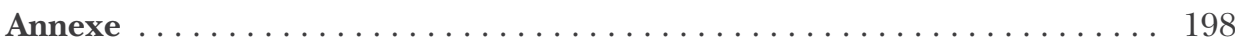

\subsection{Diffraction par rayonnement synchrotron}

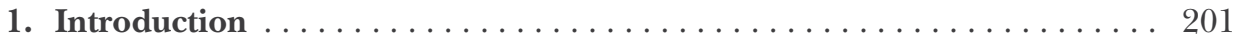

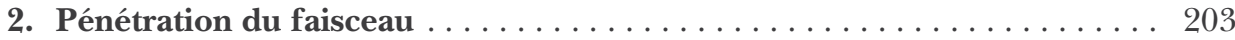

3. Volume de mesure . . . . . . . . . . . . . . . . . . . . . . . . . . 204

3.1. Géométrie en transmission et en réflexion . . . . . . . . . . . . . . . 204

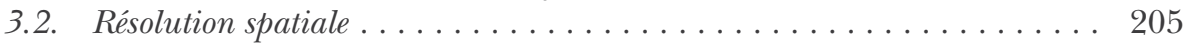

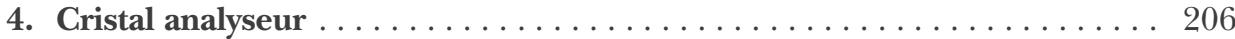


5. Position du pic de diffraction et incertitude . . . . . . . . . . . . 207

6. Installation typique à partir d'un diffractomètre poudre . . . . . . . . . 208

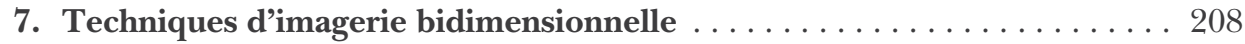

7.1. Diffraction conventionnelle 2D sur poudre . . . . . . . . . . . . . . 209

7.2. Microscopie $3 D$ par rayons X synchrotron . . . . . . . . . . . . . . 210

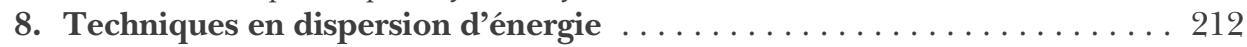

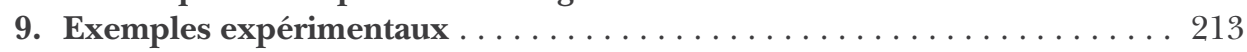

9.1. Soudage par friction malaxage analysé sur ID31 de l'ESRF . . . . . . . . . 213

9.2. Diffraction par dispersion d'énergie d'éprouvettes fissurées . . . . . . . . . . 215

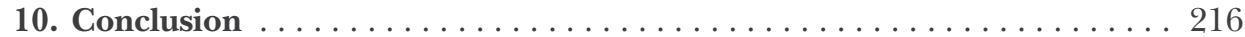

\subsection{Cartographies d'orientations et de déformations à l'échelle submicronique grâce à la microdiffraction en faisceau polychromatique}

1. Introduction . . . . . . . . . . . . . . . . . . . . . . . . . . . . . . . . 219

2. Description des composants des lignes de lumière . . . . . . . . . . 220

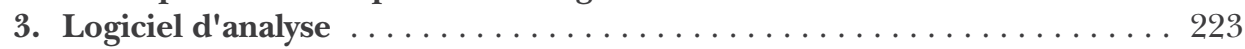

4. Exemples d'applications de la microdiffraction Laue . . . . . . . . . . 226

4.1. Échantillons à gros grains - Alliage à mémoire de forme . . . . . . . . . . 226

4.2. Échantillons à grains fins - Films minces texturés . . . . . . . . . . . . . 229

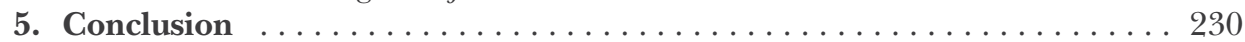

\subsection{Diffraction des rayons $X$ : un outil de choix pour l'étude des propriétés mécaniques aux petites échelles}

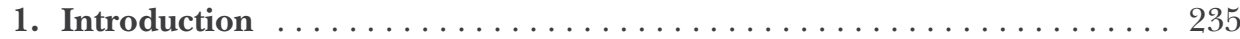

2. Diffraction cinématique des rayons $X$ : puissant outil pour l'analyse

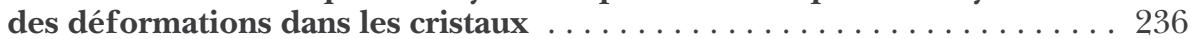

3. Faisceaux de rayons $\mathbf{X}$ de taille submicronique $\ldots \ldots \ldots \ldots \ldots \ldots$

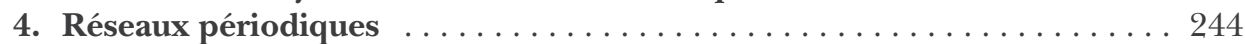

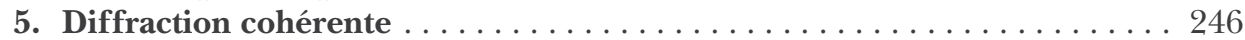

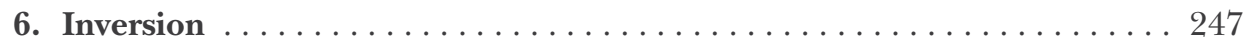

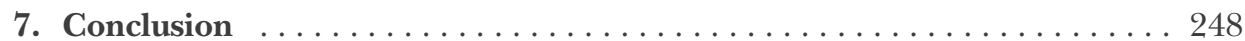

\subsection{Un regard sur l'EBSD, procédures passées et nouvelles}

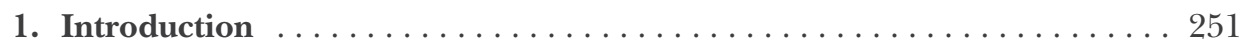

2. Principe de diffraction des électrons rétrodiffusés . . . . . . . . . . . 252

3. Formation des clichés de diffraction d'électrons rétrodiffusés . . . . . 2 255

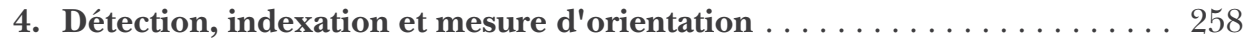

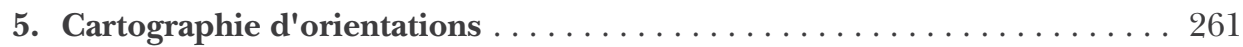

6. Cartographie de phases . . . . . . . . . . . . . . . . . 262

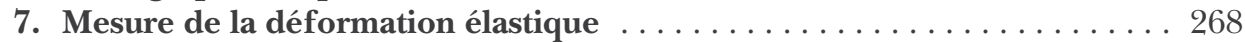

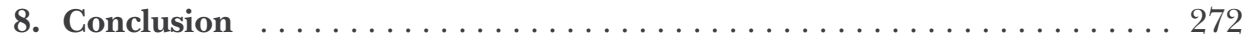




\section{Chapitre 6 : Influence de la texture}

\subsection{Représentation des orientations cristallines - Quelques exemples de texture}

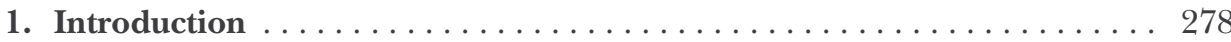

2. Description et représentation des orientations cristallines . . . . . . . . . 278

2.1. Indices de Miller, figures de pôles directes et inverses . . . . . . . . . . . . 279

2.2. Angles et espace d'Euler . . . . . . . . . . . . . . . . . . 283

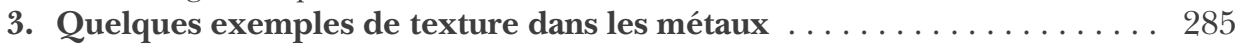

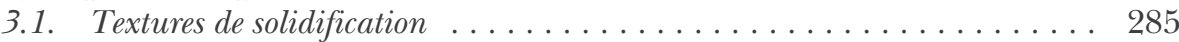

3.2. Textures de déformation à chaud . . . . . . . . . . . . . . . . . . 286

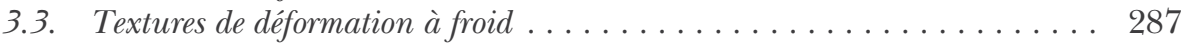

3.4. Textures de recristallisation . . . . . . . . . . . . . . . . . . . 295

4. Quelques exemples de textures (ou fabriques) dans les matériaux géologiques . . . . . . . . . . . . . . . . . . . . . . . . . . 299

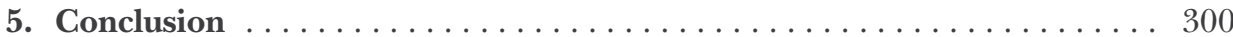

\subsection{Mesure de la texture par diffraction des rayons $X$ ou des neutrons et calcul de la FDOC}

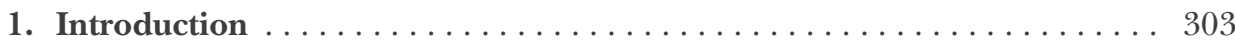

2. Mesures des figures de pôles directes par diffraction . . . . . . . . . . 304

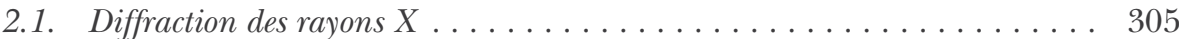

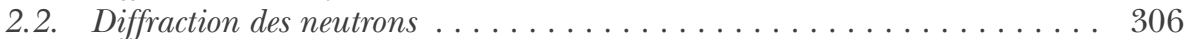

2.3. Conclusion ... . . . . . . . . . . . . . . . . . . . . . 310

3. Fonction de Distribution des Orientations Cristallines (FDOC) . . . . 311

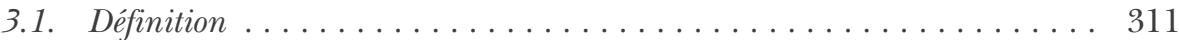

3.2. Calcul de la FDOC . . . . . . . . . . . . . . . . . . . . . . . . . 312

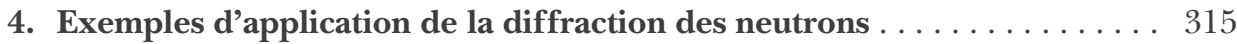

4.1. Exemple de suivi in situ de la recristallisation du cuivre . . . . . . . . . 316

4.2. Étude in situ de l'évolution de la texture de la glace
soumise à un chargement uniaxial . . . . . . . . . . . . . . . 317

4.3. Texture d'une cuirasse d'un arquebusier . . . . . . . . . . . . . . . 319

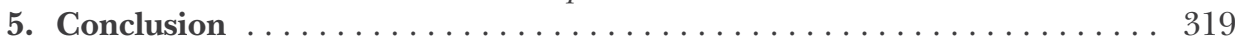

\subsection{Mesure de la texture et calcul de la FDOC à l'aide d'approches locales}

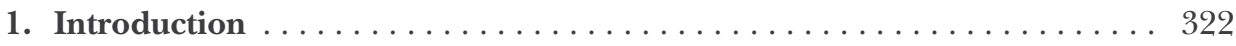

2. Méthodes de mesure des orientations cristallographiques . . . . . . . . 322

3. Corrélation entre la texture et la microstructure . . . . . . . . . . . . . . 323

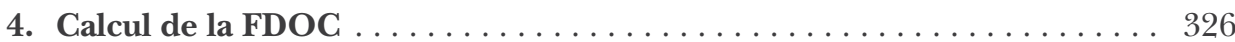

4.1. Méthode harmonique ....................... 326

4.2. Méthode de Matthies . . . . . . . . . . . . . . . . . . . . . 328

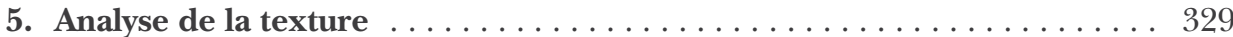

6. Conclusion ................................... 339 


\subsection{Influence des textures sur les propriétés physiques}

1. Introduction . . . . . . . . . . . . . . . . . . . . . . . . . 343

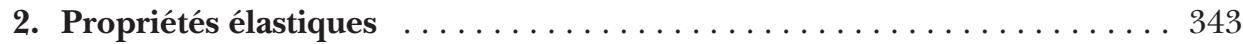

2.1. Monocristaux .......................... 344

2.2. Polycristaux ............................ 345

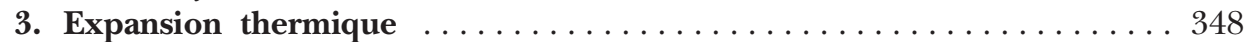

3.1. Cas des bicristaux de zinc . . . . . . . . . . . . . . . . 350

3.2. Rochet thermique (thermal ratchetting) de l'uranium . . . . . . . . . . . 352

3.3. Cas du zircaloy 2 . . . . . . . . . . . . . . . . . . . . . 354

4. Anisotropie de déformation plastique $\ldots \ldots \ldots \ldots \ldots \ldots \ldots \ldots$

4.1. Cornes d'emboutissage . . . . . . . . . . . . . . . . . . . 358

4.2. Durcissement textural (texture hardening) des matériaux de structure hexagonale compacte . . . . . . . . . . . . . . . . . . . 363

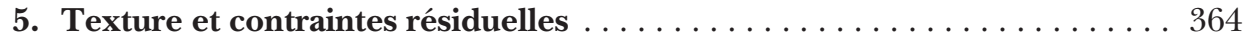

6. Texture et anisotropie magnétocristalline $\ldots \ldots \ldots \ldots \ldots \ldots \ldots \ldots$

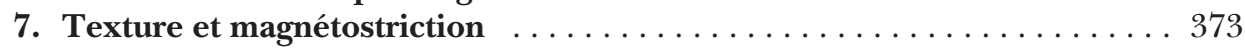

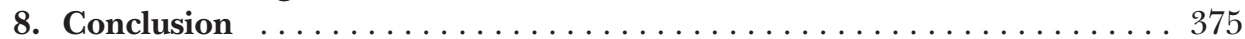

\section{Chapitre 7 : Interprétation des contraintes résiduelles à l'aide de la simulation numérique}

\subsection{Modèle autocohérent de la déformation élastoplastique et ses applications}

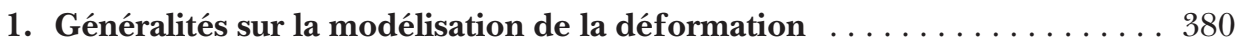

1.1. Mécanismes de la déformation plastique . . . . . . . . . . . . . . 380

1.2. Caractéristiques générales des modèles de déformation . . . . . . . . . . . 382

1.3. Notions de base . . . . . . . . . . . . . . . . . . . . . . . . . . . . . . . 384

2. Modèle autocohérent . . . . . . . . . . . . . . . . . . . . . . . . . 389

2.1. Modules tangents . . . . . . . . . . . . . . . . . . . . 389

2.2. Calcul des déformations . . . . . . . . . . . . . . . . . . . . 390

2.3. Tenseurs de concentration . . . . . . . . . . . . . . . . . . . 392

2.4. Critères de sélection des systèmes de glissement . . . . . . . . . . . . . . . . 393

3. Applications du modèle autocohérent $\ldots \ldots \ldots \ldots \ldots 393$

3.1. Prévision des textures . . . . . . . . . . . . . . . . . . . . 394

3.2. Prévision des courbes de durcissement . . . . . . . . . . . . . . . . . . . . . . 395

3.3. Interprétation des mesures des contraintes résiduelles par diffraction . . . . 395

3.4. Étude des contraintes internes dans un acier biphasé . . . . . . . . . . . . . 398

3.5. Étude des contraintes internes dans un composite AlSiC . . . . . . . . . . . . 398

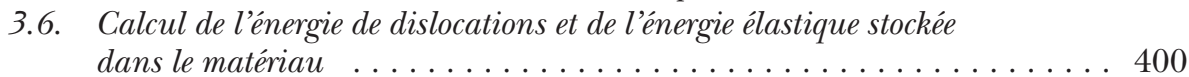

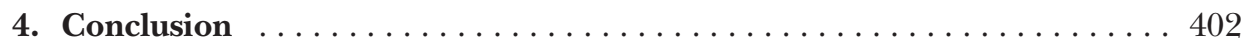


Annexe 1 Calcul des vitesses de glissement et du module tangent du grain . . . 404 Annexe 2 Équation intégrale décrivant le comportement du matériau

hétérogène

\subsection{Simulation par la méthode des éléments finis} du comportement mécanique local des polycristaux couplages physiques

1. Introduction

2. Agrégats cristallins, conditions aux limites et maillage . . . . . . . . . 410

2.1. Obtention des agrégats . . . . . . . . . . . . . . . . . 410

2.2. Maillage et conditions aux limites . . . . . . . . . . . . . . . . . . . 412

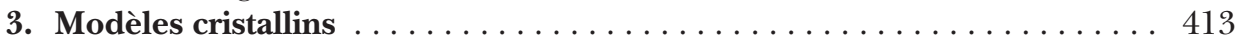

3.1. Modélisation du type forêt avec écrouissage isotrope, $T>T_{F} / 3 \ldots \ldots \ldots . .413$

3.2. Modèles du type Forêt avec écrouissage cinématique, $T>0,3 T_{F} \ldots \ldots \ldots 418$

3.3. Modèles cristallins basse température, écrouissage isotrope, $T<T_{F} / 3 \ldots \ldots 419$

3.4. Modèle de transition hautes / basses températures . . . . . . . . . . . . . . . . 424

4. Cadre de la modélisation - description du formalisme en grandes transformations ........................... 427

5. Exemples ............................ 430

5.1. Modélisation du forgeage à chaud d'un alliage de titane . . . . . . . . . . 431

5.2. Simulation de la recristallisation d'une tôle d'acier laminée Couplage déformation-recristallisation .................. 433

5.3. Simulation de la rupture en fond de fissure dans la transition fragile/ductile - Couplage déformationprobabilité de rupture . . . . . . . . . . . . . . . . . . . . 439

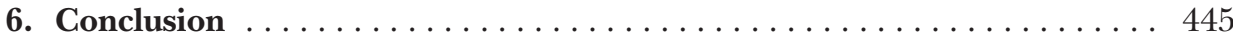

\subsection{Apport de la simulation numérique pour l'évaluation des contraintes par diffraction de neutrons et rayonnement synchrotron}

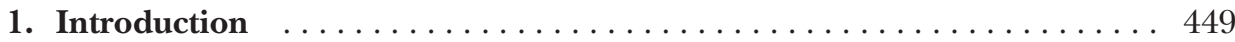

2. Simulation d'un diffractomètre de neutrons deux axes . . . . . . . . . 451

3. Évaluation des contraintes d'une pièce nitrurée . . . . . . . . . . . . 455

4. Évaluation des contraintes résiduelles d'un alliage à base nickel grenaillé 459

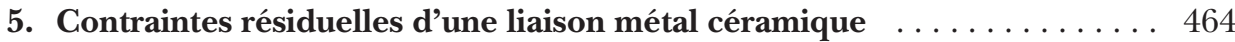

6. Contraintes résiduelles d'une soudure hétérogène . . . . . . . . . . . . . 472

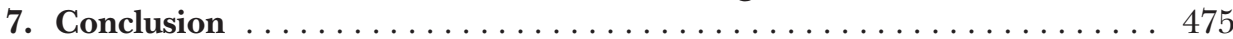

\section{Chapitre 8 : Applications}

\subsection{Applications en aéronautique}

1. Progression dans l'utilisation des grands instruments . . . . . . . . . . . 478 
2. Analyse de contraintes et corrections instrumentales . . . . . . . . . . . 479

3. Exemples d'application utilisant les grands instruments . . . . . . . . 481

3.1. Traitement de surface de nitruration . . . . . . . . . . . . . . . . . . 481

3.2. Assemblage par FSW . . . . . . . . . . . . . . . . . . . . . . . 485

3.3. Procédé de formage de tôles minces par déformations plastiques locales . . . 488

4. Conclusion . . . . . . . . . . . . . . . . . . . . . . . . . . . 491

\subsection{Mesure et modélisation de la redistribution des contraintes résiduelles, sous sollicitations cycliques, dans un assemblage fretté roue-axe ferroviaire}

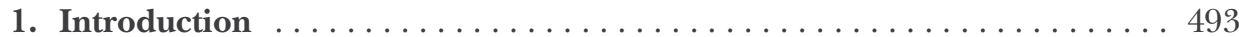

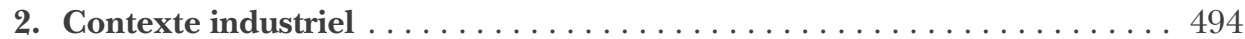

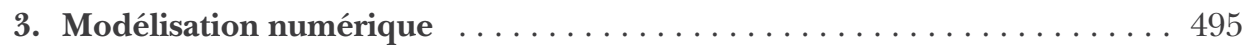

3.1. Loi de comportement du matériau . . . . . . . . . . . . . . . . . . . . . 495

3.2. Simulation numérique du calage à la presse . . . . . . . . . . . . . . . . . . 497

3.3. Simulation du chargement de fatigue . . . . . . . . . . . . . . . . . 503

3.4. Conclusion . . . . . . . . . . . . . . . . . . . . . . . . . . . . . . . . 509

4. Mise en évidence de la redistribution des contraintes résiduelles

dans l'assemblage par diffraction de neutrons . . . . . . . . . . . . 510

4.1. Étude préliminaire . . . . . . . . . . . . . . . . . . . . 510

4.2. Redistribution des champs mécaniques par fatigue oligocyclique . . . . . . . 518

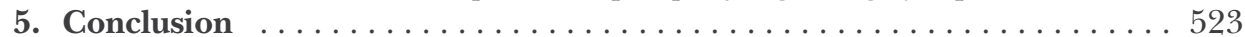

\section{3 Évaluation des contraintes résiduelles dans des assemblages soudés}

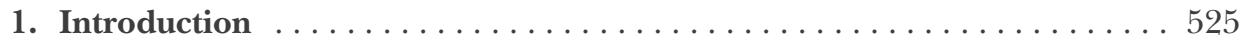

1.1. Généralités ............................. 525

1.2. Difficultés liées aux mesures de déformations dans et au voisinage de cordons de soudure . . . . . . . . . . . . . . . . . . 527

1.3. Avantages de la diffraction des neutrons sur les autres techniques permettant de déterminer des contraintes résiduelles . . . . . . . . . . . 528

2. Influence des contraintes résiduelles sur les structures mécanosoudées . . 529

2.1. Fissuration par l'hydrogène . . . . . . . . . . . . . . . . . . . . . . 529

2.2. Ruine par fatigue . . . . . . . . . . . . . . . . . . . . . . . 529

3. Exemples de mesures de déformations résiduelles par diffraction des neutrons dans le cas de structures soudées . . . . . . . . . . . . 530

3.1. Comparaison des mesures obtenues par diffraction des neutrons à d'autres techniques de mesures . . . . . . . . . . . . . . . . . . . . . 530

3.2. Quelques applications spécifiques sur les joints soudés . . . . . . . . . . 539

3.3. Comparaison entre mesures de déformations résiduelles par diffraction des neutrons et modélisations . . . . . . . . . . . . . . . . . . . . . . .544

4. Effet des textures cristallographiques sur les calculs de contraintes résiduelles dans les cordons de soudures . . . . . . . . . . . . . . . . 551

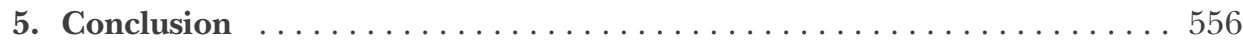




\subsection{Estimation de l'énergie stockée, force motrice de la recristallisation}

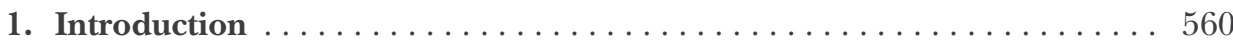

2. Mesure de l'énergie stockée en fonction de l'orientation cristallographique par diffraction . . . . . . . . . . . . . . . . . . 560

2.1. Principe de la méthode de mesure par diffraction des rayons X ou des neutrons . . . . . . . . . . . . . . . . . . . 560

2.2. Résultats obtenus par la méthode de mesure d'énergie par diffraction . . . . . 564

3. Calcul de l'énergie stockée en fonction de l'orientation cristallographique à l'aide du MET et de l'EBSD . . . . . . . . . . . . 5 571

3.1. Principe de l'approche de Dillamore . . . . . . . . . . . . . . . . . . . 571

3.2. Modification apportée à la méthode de Dillamore . . . . . . . . . . . . . 571

4. Étude comparative des mesures d'énergie . . . . . . . . . . . . . 573

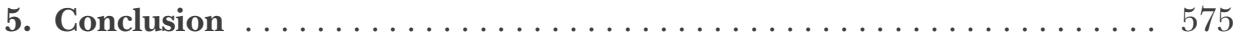

\subsection{Analyse des contraintes internes dans les matériaux composites}

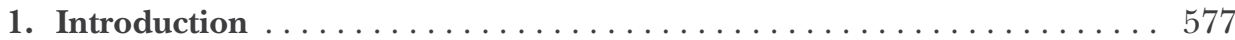

2. Les contraintes internes dans les composites $\ldots \ldots \ldots \ldots \ldots \ldots \ldots 78$

3. Application des techniques de diffraction pour l'analyse des contraintes résiduelles dans les matériaux composites . . . . . . . . . . . . . . . . . 580

3.1. Contraintes thermiques . . . . . . . . . . . . . . . . . . . 581

3.2. Contraintes induites par la déformation plastique . . . . . . . . . . . . . 582

3.3. Les contraintes générées par les différences d'élasticité . . . . . . . . . . . 583

4. Détermination des profils de macrocontraintes résiduelles ........ 585

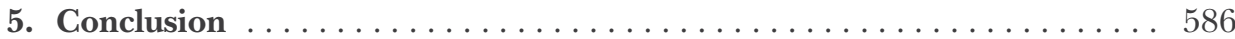

\subsection{Intérêt de la diffraction de neutrons et de rayons $X$ du rayonnement synchrotron dans l'analyse des matériaux à mémoire de forme}

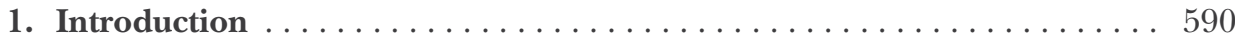

2. Mesure des déformations et analyse des contraintes par diffraction de neutrons . . . . . . . . . . . . . . . . . . . . . . . . . . 590

2.1. Diffraction de neutrons en temps de vol-Études expérimentales sur les alliages FePd, CuAlZnMn et NiTi . . . . . . . . . . 590

2.2. Diffraction de neutrons à haute résolution en angleEtude expérimentale sur l'alliage CuAlBe . . . . . . . . . . . . . 593

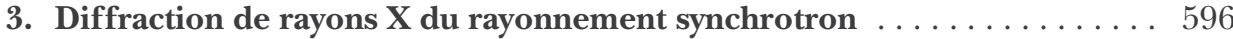

3.1. Le microscope $3 D X R D \ldots \ldots \ldots \ldots \ldots \ldots \ldots \ldots$. . . . . . . . . . . 597

3.2. Technique "MicroLaue» ou microdiffraction polychromatique . . . . . . . 601

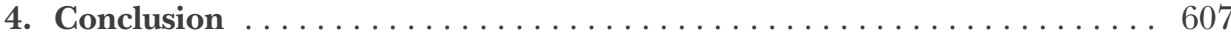

\subsection{Caractérisation des biomatériaux implantaires}

1. Introduction ...................................... 609 


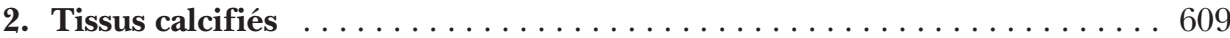

2.1. Le tissu osseux . . . . . . . . . . . . . . . . . . . . . . . . . . . . . . 610

2.2. Mesures expérimentales dans l'os . . . . . . . . . . . . . 610

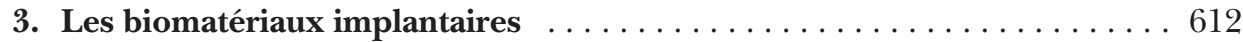

3.1. Modélisation de la projection d'HAp par torche plasma sur substrat de titane . . . . . . . . . . . . . . . . . . . . . . 613

3.2. Contraintes résiduelles déterminées par la méthode de diffraction de rayonnement synchrotron . . . . . . . . . . . . . . . . 615

4. Interface os-implant en titane revêtu par HAp . . . . . . . . . . . . . . 619

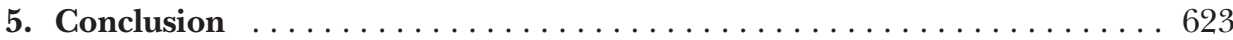

\subsection{Déformations résiduelles dans les géomatériaux : exemple des roches riches en quartz}

1. Introduction

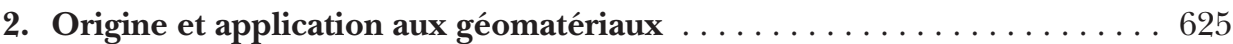

3. Classification des déformations ou des contraintes résiduelles . . . . . . 626

4. Méthodes de diffraction appliquées aux géomatériaux . . . . . . . . . . . 629

5. Déformations résiduelles dans des roches faiblement déformées à froid : les grès

6. Cas d'études des déformations résiduelles par des méthodes distinctes de la diffraction des neutrons . . . . . . . . . . . . . . . . . . . 633

6.1. Exemple du plissement d'une couche de grès . . . . . . . . . . . . . . 633

6.2. Déformation de plaques de marbre . . . . . . . . . . . . . . . . . . . . 634

7. Hétérogénéité des déformations résiduelles dans les géomatériaux . . . 636

8. Les déformations résiduelles dans les quartzites :

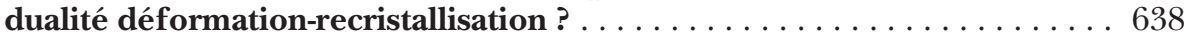

9. Interprétation du tenseur des déformations résiduelles.

Application à la modélisation numérique des textures . . . . . . . . . . . . . 642

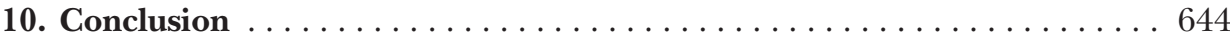

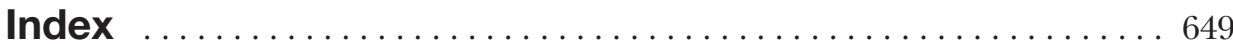


This page intentionally left blank 